\title{
CONTRAST-ENHANCED PHOTOACOUSTIC IMAGING USING INDOCYANINE GREEN-CONTAINING NANOPARTICLES
}

\author{
JUNPING ZHONG and SIHUA YANG* \\ MOE Key Laboratory of Laser Life Science and \\ Institute of Laser Life Science \\ South China Normal University \\ Guangzhou 510631, P. R. China \\ *yangsh@scnu.edu.cn
}

Received 4 June 2013

Accepted 1 July 2013

Published 31 July 2013

\begin{abstract}
Contrast agents are attracting a great deal of attention in photoacoustic imaging. Here we introduce an exogenous contrast agent that provides high photoacoustic signal amplitude at the near-infrared wavelength. Our agents consist of Indocyanine green (ICG) and phospholipid-polyethylene glycol (PL-PEG), entitled ICG-PL-PEG nanoparticles. These nanoparticles have overcome numerous limitations of ICG, such as poor aqueous stability, concentrationdependent aggregation and lack of target specificity. ICG-PL-PEG nanoparticles are biocompatible and relatively nontoxic. All the components of ICG-PL-PEG nanoparticles have been approved for human use. Upon pulsed laser irradiation, the nanoparticles are more efficient in producing photoacoustic waves than ICG alone. The results showed that ICG-PL-PEG nanoparticles act as good contrast agents for photoacoustic imaging. These unique ICG-PL-PEG nanoparticles have great potential in clinical applications.
\end{abstract}

Keywords: Photoacoustic imaging; nanoparticle; indocyanine green; phospholipid-polyethylene glycol.

\section{Introduction}

Photoacoustic imaging is a noninvasive imaging method with good resolution and high contrast in a sufficient depth range. ${ }^{1-3}$ In photoacoustic imaging, when the absorbers are irradiated by pulsed lasers, some of the light is absorbed and result in a rapid thermoelastic expansion. Then the photoacoustic waves will be generated. The photoacoustic waves can be detected by a highly sensitive ultrasound transducer, and thus used to spatially resolve the location of absorbers. ${ }^{4}$ Photoacoustic imaging applications range from microscopy to whole organ imaging. It has been successfully applied in early detection of breast tumor, ${ }^{5}$ brain functional imaging, ${ }^{6}$ noninvasive monitoring of cerebrovascular activities and blood oxygenation ${ }^{7}$ and monitoring of vascular damage during tumor photodynamic therapy. ${ }^{8}$

This is an Open Access article published by World Scientific Publishing Company. It is distributed under the terms of the Creative Commons Attribution 3.0 (CC-BY) License. Further distribution of this work is permitted, provided the original work is properly cited. 
Indocyanine green (ICG) is popular in photoacoustic methods, since it is currently the only Federal Drug Administration (FDA) approved nearinfrared (NIR) clinical imaging agent that exhibits extraordinarily high optical adsorption. ${ }^{9}$ However, applications of ICG remain limited by its poor aqueous stability, concentration-dependent aggregation, lack of target specificity and quick clearance from the body with a short half-life about $2-4$ min. ${ }^{10-18}$ To overcome these limitations, ICGPL-PEG nanoparticles consisting of ICG and phospholipids-polyethylene glycol (PL-PEG) was developed as photoacoustic contrast agents. The nanoparticles provide multiple advantages. ICGPL-PEG nanoparticles are biocompatible and relatively nontoxic. ICG-PL-PEG nanoparticles can improve the molecular stability of ICG and prolong its plasma half-life. ICG-PL-PEG nanoparticles are also small enough to avoid clearance by the reticuloendothelial system and permeation into tumor tissue. The hydrophilic PEG surrounding the nanoparticles surface renders them sterically stable, protecting them from mononuclear phagocytic system uptake. ${ }^{17-19}$ The absorption cross section of myriad nanoparticles can be multiples to orders of magnitude higher than the surrounding native tissue at NIR wavelengths. They are ideally suited as photoacoustic contrast agents.

In this work, ICG-PL-PEG nanoparticles were developed utilizing the noncovalent self-assembly chemistry between PL-PEG and ICG. The properties of ICG-PL-PEG nanoparticles, such as absorption spectra, stability, morphology and size distribution, were investigated. The feasibility of using these nanoparticles as photoacoustic imaging contrast agents was evaluated. Preliminary results showed that a high contrast in mice could be achieved.

\section{Materials and Methods}

\subsection{Materials}

ICG for injection was purchased from Dan Dong Yi Chuang Company (Dandong, Liaoning, China). 1,2-distearoyl-sn-glycero-3-phosphoethanol-amineN-[amino/carboxy(PEG)2000] was purchased from Avanti Polar Lipids Inc. (AL, USA) and other chemicals used in this work were all of analytical grade. All of the reagents were used without further purification.

\subsection{Preparation of ICG-PL-PEG nanoparticles}

Preparation of ICG-PL-PEG nanoparticles was performed following the procedures according to Zheng et al. ${ }^{17}$

\subsection{Characterization experiments}

The absorption spectra of ICG solution and ICG-PL-PEG nanoparticles were investigated by a UV/Vis spectrometer (Lambda 35, Perkin-Elmer, MA, USA). The morphology and size of the ICG-PL-PEG nanoparticles were investigated by A JEM-100CXII (Jeol Inc., Tokyo, Japan) transmission electron microscope (TEM) with parameters of $100 \mathrm{kV}$ voltage and $70 \mathrm{pA}$ current.

\subsection{Photoacoustic system}

The photoacoustic device was designed according to Cheng et al. ${ }^{20}$ An optical parametric oscillator (OPO) (VIBRANT B 532I, OPOTEK, USA) with a full-width at a high magnitude of $10 \mathrm{~ns}$ and a repetition of $10 \mathrm{~Hz}$ was used as the light source. For all studies, the maximum laser energy per pulse was $10 \mathrm{~mJ} / \mathrm{cm}^{2}$. A focused ultrasound transducer with center frequency of $15 \mathrm{MHz}$ and $-6 \mathrm{~dB}$ bandwidth of $100 \%$ was used to receive the photoacoustic signals generated by the tested sample on the threedimensional (3D) scanning stage driven by computer-controlled stepper motors. The photoacoustic signals were recorded by the computer through the signal amplifier and a dual-channel data acquisition card. The sampling rate of the data acquisition card

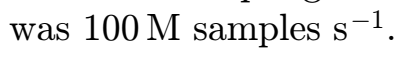

\section{Results}

\subsection{Characterization of the ICG-PL-PEG nanoparticles}

The PEG-coated, amine-functionalized base ICGPL-PEG nanoparticles are designed to utilize the noncovalent self-assembly chemistry between ICG and PL-PEG. The absorption spectrum of freely dissolved ICG $(5 \mu \mathrm{g} / \mathrm{ml})$ and ICG-PL-PEG solution (containing $5 \mu \mathrm{g} / \mathrm{ml} \mathrm{ICG}$ ) are shown in Fig. 1(a). The main peaks of the absorption of ICG-PL-PEG shift approximately $20 \mathrm{~nm}$ toward the higher wavelengths compared with freely dissolved ICG. A TEM 


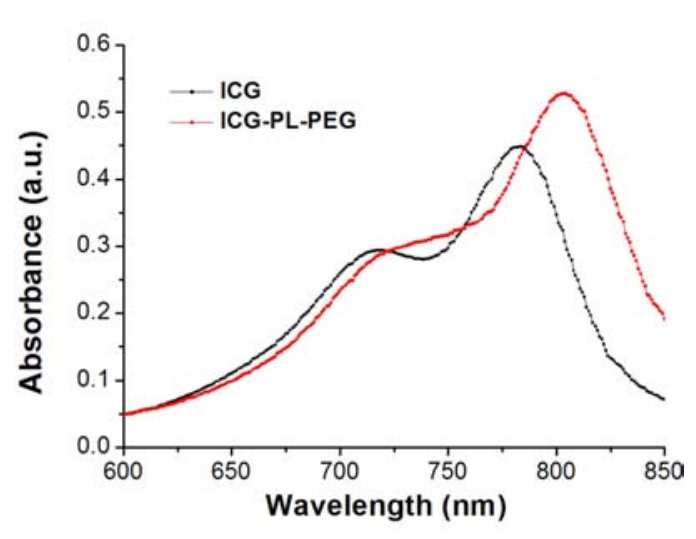

(a)

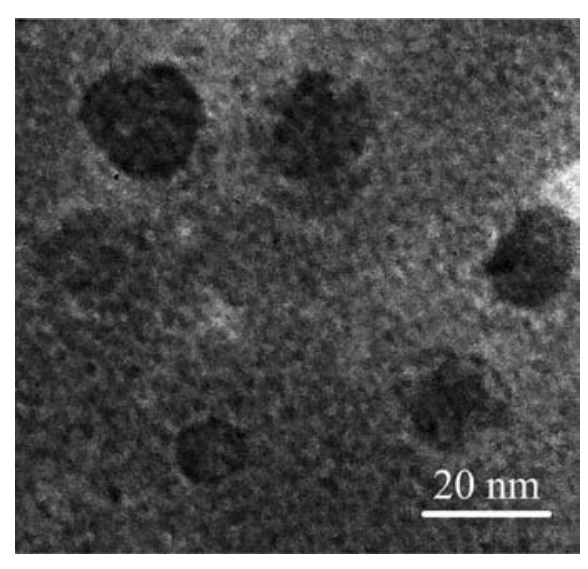

(b)

Fig. 1. (a) Absorption spectra of ICG and ICG-PL-PEG nanoparticles, respectively. (b) TEM imaging of ICG-PL-PEG nanoparticles.

image of the ICG-PL-PEG nanoparticles [see Fig. 1(b)] showed that the nanoparticles were spherical and well dispersed, with a core size of approximately $18 \mathrm{~nm}$. The photoacoustic signal generated by ICG and ICG-PL-PEG nanoparticles at various concentrations was detected using a pulsed laser as the irradiation source. The choice of laser wavelength was based on the absorbance spectra of ICG and ICG-PL-PEG nanoparticles. The photoacoustic signals generated by the ICG-PL-PEG nanoparticles are summarized in Fig. 2. The intensity of the photoacoustic signal produced by ICGPL-PEG and ICG was observed to be linearly dependent on the concentrations. The intensity of the photoacoustic signal produced by ICGPL-PEG was much stronger than ICG at the same concentration.

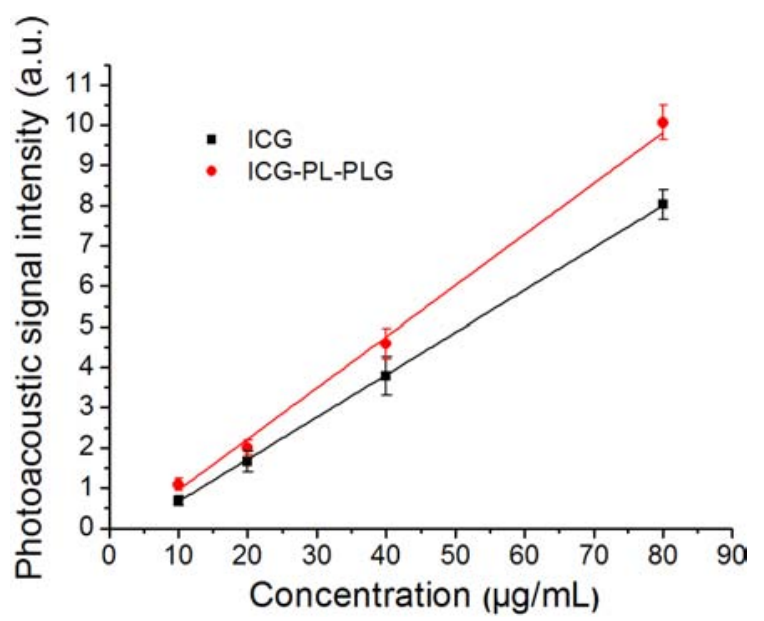

Fig. 2. Photoacoustic signals of ICG and ICG-PL-PEG nanoparticles at various concentrations.

\subsection{Stability of freely dissolved ICG and ICG-PL-PEG nanoparticles}

We measured absorption spectra of the ICG $(5 \mu \mathrm{g} /$ $\mathrm{ml}$ ) and ICG-PL-PEG nanoparticles (containing $5 \mu \mathrm{g} / \mathrm{ml} \mathrm{ICG)}$ at different times. The samples were maintained at room temperature without light exposure. The absorbance of the ICG was almost completely degraded after 40 days [see Fig. 3(a)]. However, the ICG-PL-PEG nanoparticles only experienced a slight reduction of absorbance after 40 days [see Fig. 3(b)]. These experiments demonstrated that the nanoparticles had a higher stability than freely dissolved ICG.

\subsection{Photoacoustic imaging of the ICG-PL-PEG nanoparticles}

Four identical plastic tubes with a diameter of $3.2 \mathrm{~mm}$ that filled with different concentrations of ICG-PL-PEG solution were imaged by photoacoustic imaging. Four tubes were filled with ICG-PL-PEG containing 10, 20, 40, $80 \mu \mathrm{g} / \mathrm{ml} \mathrm{ICG}$ respectively. A high-contrast photoacoustic imaging can be reconstructed with high signal-noise-ratio (SNR) photoacoustic signals. As shown in Fig. 4(a), when the concentration of ICG-PL-PEG increased, the SNR of the absorber imaging increased accordingly, which clearly demonstrated the advantage of ICG-PL-PEG enhancement. Photoacoustic imaging of the samples resulted in a visible increase in signal as the concentration of ICG-PL-PEG nanoparticles increased, as shown in Fig. 4(b). The result indicated that $\mathrm{ICG}-\mathrm{PL}-\mathrm{PEG}$ nanoparticles 


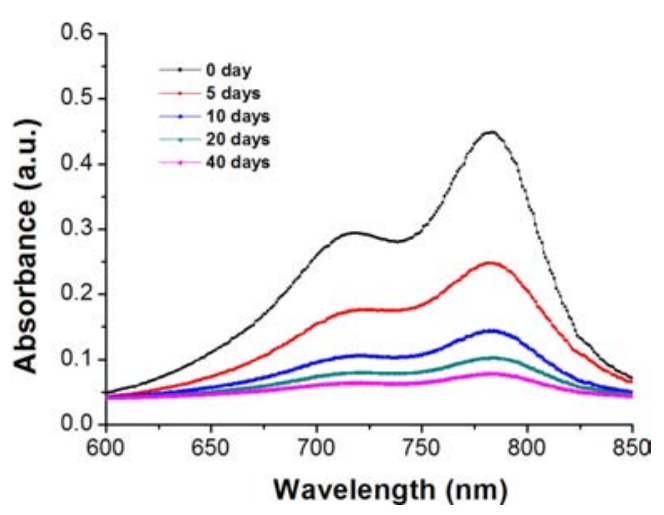

(a)

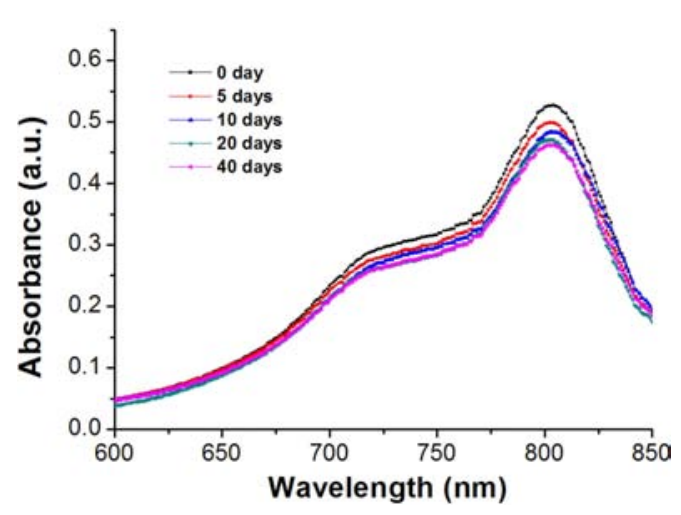

(b)

Fig. 3. Absorption of ICG (a) and ICG-PL-PEG nanoparticles (b) at different times.

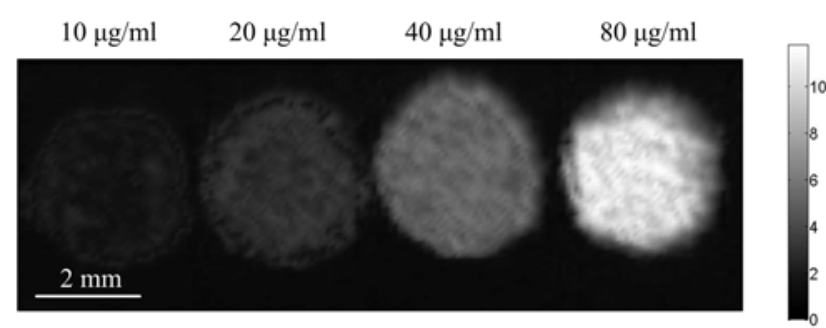

(a)

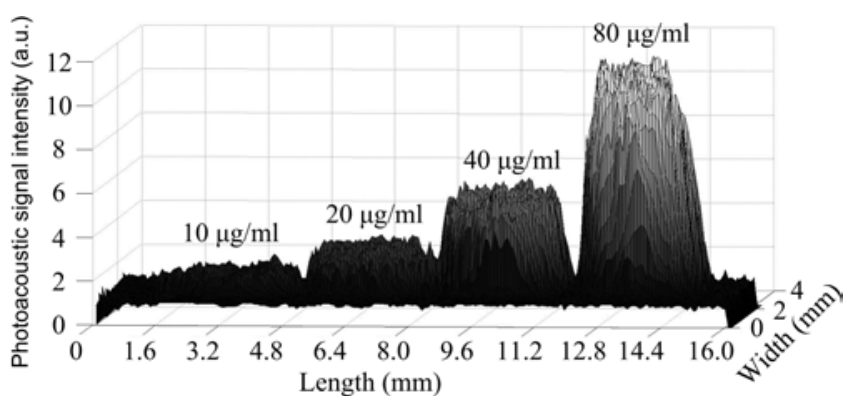

(b)

Fig. 4. (a) Photoacoustic imaging of ICG-PL-PEG nanoparticles at four different concentrations in plastic tubes with a diameter of $3.2 \mathrm{~mm}$. (b) Photoacoustic signals of the ICG-PL-PEG nanoparticles at different concentrations correspond to Fig. 4(a).

have a potential to improve tissue contrast in photoacoustic imaging.

One of the advantages of photoacoustic imaging is its ability to detect nanoparticles in tissue. This capability was tested by performing photoacoustic imaging on mice. Approximately $50 \mu \mathrm{l}$ ICGPL-PEG solution (containing $0.1 \mathrm{mg} / \mathrm{ml} \mathrm{ICG)} \mathrm{and}$ $0.1 \mathrm{mg} / \mathrm{ml}$ ICG solution were injected into the flank

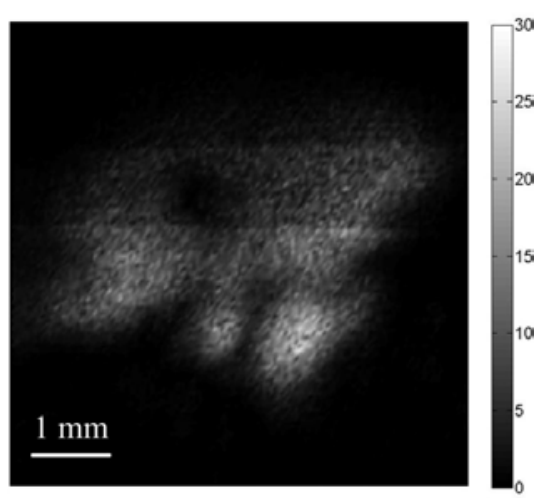

(a)

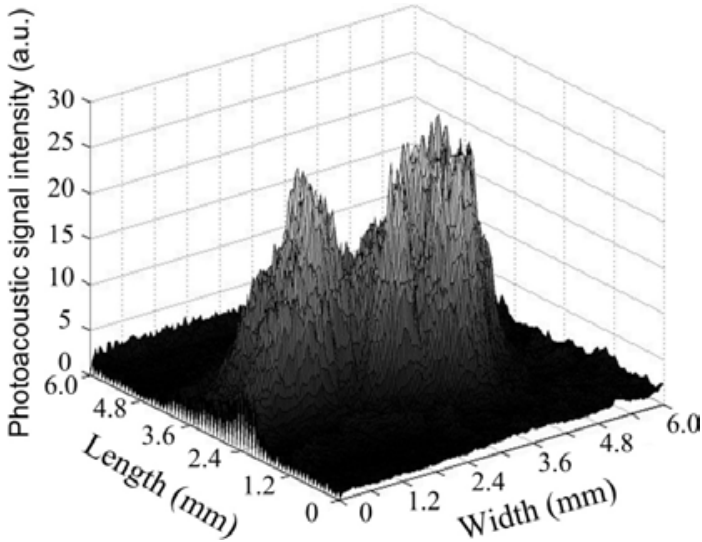

(b)

Fig. 5. Photoacoustic imaging (a) and corresponding signals (b) of ICG-PL-PEG nanoparticles. Photoacoustic imaging (c) and corresponding signals (d) of ICG. The ICG-PL-PEG nanoparticles and ICG were injected directly into the flank region of female $\mathrm{Balb} / \mathrm{c}$ mice. 


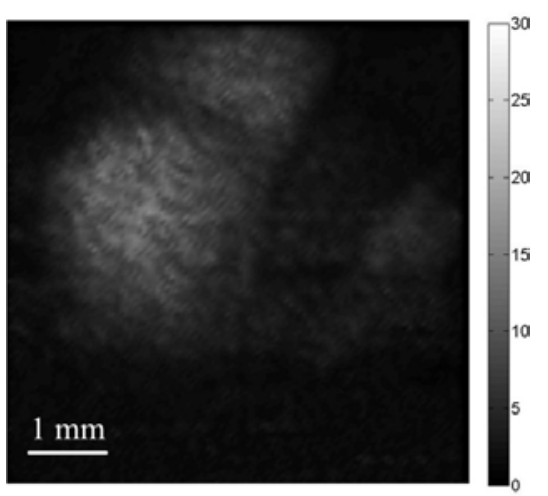

(c)

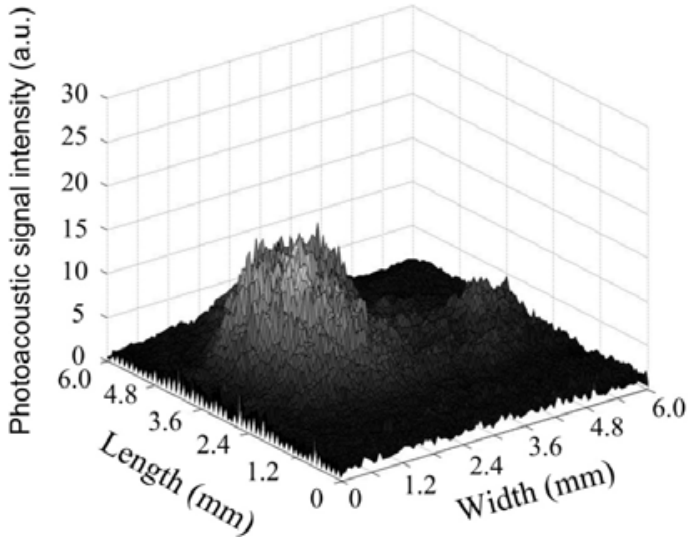

(d)

Fig. 5. (Continued)
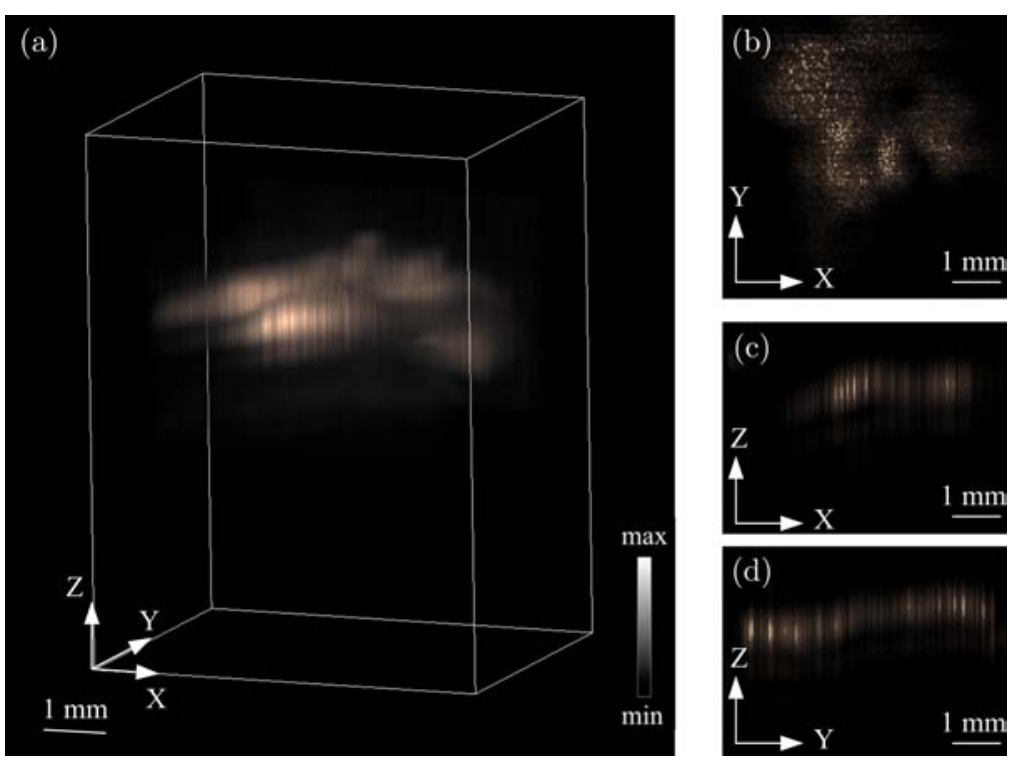

Fig. 6. (a) 3D photoacoustic imaging of ICG-PL-PEG nanoparticles injected directly into the flank region of female Balb/c mice, (b) Three slices of two-dimensional photoacoustic images correspond to Fig. 6(a).

region of female Balb/c mice, respectively. Then, photoacoustic imaging was performed. The contrast results for imaging experiments between the dissolved ICG and ICG-PL-PEG particles in the biological tissues show the performance of the ICG-PL-PEG particles [see Figs. 5(a) and 5(b)] is better than that of pure ICG [see Figs. 5(c) and 5(d)]. The 3D photoacoustic image [see Fig. 6(a)] shows the signal received primarily from the ICG-PL-PEG nanoparticles interrogated by the pulse of laser light. Figures 6(a)-6(d) clearly depict the location of the ICG-PL-PEG nanoparticles against the background tissue. These images demonstrate the ability of photoacoustic imaging to locate accumulated nanoparticles inside the tissue. Thus, if the ICGPL-PEG nanoparticles were injected in the bloodstream systematically and accumulated in tumor, then photoacoustic imaging could be used to locate the nanoparticles inside the tissue, helping clinicians to better define and characterize diseased areas.

\section{Discussion}

The photoacoustic technique has a broad range of applications, ${ }^{21-23}$ including nondestructive biomedical imaging, chemical analysis and ecology. The acoustic waves are excited when pulsed light is absorbed by a target. There are four mechanisms of 
laser-generated acoustic waves, including thermoelastic expansion, vaporization, photochemical processes and optical breakdown. ${ }^{24,25}$

The amplitude of the photoacoustic wave depends on both the optical absorption and the properties of the target. Contrast agents with strong optical absorption could significantly enhance the photoacoustic effect. ${ }^{26}$ As an FDA-approved NIR dye, ICG has been used for photoacoustic imaging. However, numerous disadvantageous physicochemical characteristics, such as poor aqueous stability, concentration-dependent aggregation and lack of target specificity, limit the application of ICG. ${ }^{16}$ Furthermore, ICG binds almost completely (98\%) to plasma albumin, leading to its low vascular or tissue permeability. ${ }^{27}$ ICG-PL-PEG nanoparticles overcome these limitations. All of the components of the nanoparticles are biocompatible and relatively nontoxic. PEG is also FDA approved for biomedical imaging and therapy. The addition of PEG provides several advantages. It can render the nanoparticles stable and compatible with biological media. It also can reduce uptake by the reticuloendothelial system and to provide the nanoparticles with stealth properties for longer circulation times in vivo. ${ }^{28-32}$

ICG-PL-PEG nanoparticles provide three mechanisms of contrast enhancement than ICG. Firstly high concentration of ICG loaded in the nanoparticles. Secondly encapsulation in nanoparticles stabilizes ICG against an aqueous media and other destabilizing effects from the biological environment. Lastly ICG-PL-PEG nanoparticles have more efficient light absorption than ICG at the maximum absorption (with the same ICG concentration). ICG-PL-PEG nanoparticles have been demonstrated to produce high levels of contrast enhancement both in phantom and live animal experiments, confirming the feasibility of the ICGPL-PEG nanoparticles as contrast agents for clinical photoacoustic imaging. As such, the ICGPL-PEG nanoparticles are naturally applicable for photoacoustic imaging.

The ICG-PL-PEG nanoparticles provide photoacoustic contrast enhancement. ICG-PL-PEG nanoparticles are specifically designed to be used in biological contrast-enhancing applications. Beyond the use as contrast agents, ICG-PL-PEG nanoparticles are adaptable for a variety of applications ranging from fundamental biomedical studies to medical diagnostic and therapeutic applications. ${ }^{17-19}$

\section{Conclusion}

In summary, the ability of ICG-PL-PEG nanoparticles for photoacoustic imaging was demonstrated. ICG-PL-PEG nanoparticles have a highly stable structure and functionality in light absorption. ICG-PL-PEG nanoparticles have high optical extinction at NIR wavelengths. All the components of ICG-PL-PEG nanoparticles are nontoxic. The experiment results turned out that ICG-PL-PEG nanoparticles can be used as contrast agents for photoacoustic imaging, which would be suitable for delineation of tumor.

\section{Acknowledgments}

This research is supported by the National Basic Research Program of China (2011CB910402; 2010CB732602), the Program for Changjiang Scholars and Innovative Research Team in University (IRT0829), the National Natural Science Foundation of China $(81127004,11104087)$, the Foundation for Distinguished Young Talents in Higher Education of Guangdong, China (LYM10061), the Specialized Research Fund for the Doctoral Program of Higher Education (20114407120001) and the Science and Technology Project of Guangzhou, China (2012J4100114).

\section{References}

1. D. Yang, D. Xing, H. Gu, Y. Tan, L. Zeng, "Fast multi-element phase-controlled photoacoustic imaging based on limited-field filtered back projection algorithm," Appl. Phys. Lett. 87, 194101 (2005).

2. B. Yin, D. Xing, Y. Wang, Y. Zeng, Y. Tan, Q. Chen, "Fast photoacoustic imaging system based on 320-element linear transducer array," Phys. Med. Biol. 49, 1339-1346 (2004).

3. Y. G. Zeng, D. Xing, Y. Wang, B. Z. Yin, Q. Chen, "Photoacoustic and ultrasonic coimage with a linear transducer array," Opt. Lett. 29, 1760-1762 (2004).

4. Y. Yuan, S. H. Yang, D. Xing, "Optical-resolution photoacoustic microscopy based on two-dimensional scanning galvanometer," Appl. Phys. Lett. 100, $023702(2012)$.

5. S. A. Ermilov, T. Khamapirad, A. Conjusteau, M. H. Leonard, R. Lacewell, K. Mehta, T. Miller, A. A. Oraevsky, "Laser optoacoustic imaging system for detection of breast cancer," J. Biomed. Opt. 14, 024007 (2009).

6. S. Yang, D. Xing, Q. Zhou, L. Xiang, Y. Lao, "Functional imaging of cerebrovascular activities in 
small animals using high-resolution photoacoustic tomography," Med. Phys. 34, 3294-3301 (2007).

7. H. F. Zhang, K. Maslov, M. Sivaramakrishnan, G. Stoica, L. H. V. Wang, "Imaging of hemoglobin oxygen saturation variations in single vessels in vivo using photoacoustic microscopy," Appl. Phys. Lett. 90, 053901 (2007).

8. L. Z. Xiang, D. Xing, H. M. Gu, D. W. Yang, S. H. Yang, L. M. Zeng, W. R. Chen, "Real-time optoacoustic monitoring of vascular damage during photodynamic therapy treatment of tumor," J. Biomed. Opt. 12, 014001 (2007).

9. C. Kim, K. H. Song, F. Gao, L. V. Wang, "Sentinel lymph nodes and lymphatic vessels: Noninvasive dual-modality in vivo mapping by using indocyanine green in rats-volumetric spectroscopic photoacoustic imaging and planar fluorescence imaging," Radiology 255, 442-450 (2010).

10. V. B. Rodriguez, S. M. Henry, A. S. Hoffman, P. S. Stayton, X. Li, S. H. Pun, "Encapsulation and stabilization of indocyanine green within poly(styrene-alt-maleic anhydride) block-poly(styrene) micelles for near-infrared imaging," J. Biomed. Opt. 13, 014025 (2008).

11. V. Saxena, M. Sadoqi, J. Shao, "Enhanced photostability, thermal-stability and aqueous-stability of indocyanine green in polymeric nanoparticulate systems," J. Photochem. Photobiol. B 74, 29-38 (2004).

12. V. Saxena, M. Sadoqi, J. Shao, "Indocyanine greenloaded biodegradable nanoparticles: Preparation, physicochemical characterization and in vitro release," Int. J. Pharm. 278, 293-301 (2004).

13. R. X. Xu, J. W. Huang, J. S. Xu, D. X. Sun, G. H. Hinkle, E. W. Martin, S. P. Povoski, "Fabrication of indocyanine green encapsulated biodegradable microbubbles for structural and functional imaging of cancer," J. Biomed. Opt. 14, 034020 (2009).

14. J. R. Rajian, M. L. Fabiilli, J. B. Fowlkes, P. L. Carson, "Drug delivery monitoring by photoacoustic tomography with an ICG encapsulated double emulsion," Opt. Express 19, 14335-14347 (2011).

15. K. Mitra, J. Melvin, S. F. Chang, K. Park, A. Yilmaz, S. Melvin, R. X. Xu, "Indocyanine-green-loaded microballoons for biliary imaging in cholecystectomy," J. Biomed. Opt. 17(11), 116025 (2012).

16. R. C. Benson, H. A. Kues, "Fluorescence properties of indocyanine green as related to angiography," Phys. Med. Biol. 23, 159-163 (1978).

17. X. Zheng, D. Xing, F. Zhou, B. Wu, W. R. Chen, "Indocyanine green-containing nanostructure as near infrared dual-functional targeting probes for optical imaging and photothermal therapy," Mol. Pharm. 8, 447-456 (2011).

18. X. Zheng, F. Zhou, B. Wu, W. R. Chen, D. Xing, "Enhanced tumor treatment using biofunctional indocyanine green-containing nanostructure by intratumoral or intravenous injection," Mol. Pharm. 9, 514-522 (2012).

19. J. Zhong, S. Yang, X. Zheng, T. Zhou, D. Xing, "In vivo photoacoustic therapy with cancer-targeted indocyanine green-containing nanoparticles," Nanomedicine, 8, 903-919 (2012).

20. Z. Chen, S. Yang, D. Xing, "In vivo detection of hemoglobin oxygen saturation and carboxyhemoglobin saturation with multiwavelength photoacoustic microscopy," Opt. Lett. 37, 3414-3416 (2012).

21. L. Silvestri, A. L. A. Mascaro, J. Lotti, L. Sacconi, F. S. Pavone, "Advanced optical techniques to explore brain structure and function," J. Innov. Opt. Health. Sci. 6, 1230002 (2013).

22. Z. Ji, Y. Fu, S. H. Yang, "Microwave-induced thermoacoustic imaging for early breast cancer detection," J. Innov. Opt. Health. Sci. 6, 1350001 (2013).

23. T. Jetzfellner, V. Ntziachristos, "Performance of blind deconvolution in optoacoustic tomography," J. Innov. Opt. Health. Sci. 4, 385-393 (2011).

24. H. X. Chen, G. Diebold, "Chemical generation of acoustic waves: A giant photoacoustic effect," Science 270, 963-966 (1995).

25. K. Wilson, K. Homan, S. Emelianov, "Biomedical photoacoustics beyond thermal expansion using triggered nanodroplet vaporization for contrastenhanced imaging," Nat. Commun. 3, 618 (2012).

26. Y. S. Chen, W. Frey, S. Aglyamov, S. Emelianov, "Environment-Dependent generation of photoacoustic waves from plasmonic nanoparticles," Small 8, 47-52 (2012).

27. S. Yoneya, T. Saito, Y. Komatsu, I. Koyama, K. Takahashi, J. Duvoll-Young, "Binding properties of indocyanine green in human blood," Invest. Ophthalmol. Vis. Sci. 39, 1286-1290 (1998).

28. F. Zhou, S. Wu, B. Wu, W. R. Chen, D. Xing, "Mitochondria-targeting single-walled carbon nanotubes for cancer photothermal therapy," Small 7, 2727-2735 (2011).

29. F. Zhou, D. Xing, B. Wu, S. Wu, Z. Ou, W. R. Chen, "New insights of transmembranal mechanism and subcellular localization of noncovalently modified single-walled carbon nanotubes," Nano Lett. 10, 1677-1681 (2010).

30. J. V. Jokerst, T. Lobovkina, R. N. Zare, S. S. Gambhir, "Nanoparticle PEGylation for imaging and therapy," Nanomedicine 6, 715-728 (2011).

31. F. Zhou, D. Xing, Z. Ou, B. Wu, D. E. Resasco, W. R. Chen, "Cancer photothermal therapy in the near-infrared region by using single-walled carbon nanotubes," J. Biomed. Opt 14, 021009 (2009).

32. D. Yu, A. Wang, H. Huang, Y. Chen, "PEG-PBLG nanoparticle-mediated HSV-TK/GCV gene therapy for oral squamous cell carcinoma," Nanomedicine $\mathbf{3}$, 813-821 (2008). 